NEC 32.2 (Vogelbaum) Glioblastoma, Part I: Surgical Management and Adjuncts

\title{
Surgery For Glioblastoma in Elderly Patients.
}

Marco Conti Nibali ${ }^{1,2}$ M.D., Lorenzo G. Gay ${ }^{1,2}$ M.D., Tommaso Sciortino ${ }^{1,2}$ M.D., Marco Rossi ${ }^{1,2}$ M.D., Manuela Caroli ${ }^{2}$ M.D., Lorenzo Bello $^{1,2}$ M.D. and Marco Riva ${ }^{2,3}$ M.D

1. Department of Oncology and Hemato-Oncology, Via Festa del Perdono 7, 20122, Milan, ITALY.

2. Unit of Neurosurgery, Fondazione IRCCS Ca' Grande Ospedale Maggiore Policlinico, Milan, ITALY.

3. Department of Medical Biotechnology and Translational Medicine, Università degli Studi di Milano, Via Festa del Perdono 7, 20122, Milan, ITALY.

Marco Conti Nibali ${ }^{1,2}$ M.D.; marco.continibali@gmail.com; @_Dr_mcn

Lorenzo G. Gay ${ }^{1,2}$ M.D.; lorenzogabrielgg@gmail.com;

Tommaso Sciortino ${ }^{1,2}$ M.D.; tsciortino11@gmail.com

Marco Rossi ${ }^{1,2}$ M.D.; rossi.marco@gmail.com 
Manuela Caroli² M.D.; manuela.caroli@policlinico.mi.it

Lorenzo Bello ${ }^{1,2}$ M.D.; lorenzo.bello@unimi.it

Marco Riva ${ }^{2,3}$ M.D.; marco.riva@unimi.it; @mriva_eu

\section{Corresponding Author}

Marco Conti Nibali, M.D.; marco.continibali@gmail.com

\section{Disclosure Statement}

The Authors have nothing to disclose.

Key Words GBM; Elderly; resection; adjuvant treatment; elderly patients; Radiotherapy; Chemoterapy; Survival

\section{Key Points}

- The incidence of GBM increases with age, with highest rate in the population between 75 and 84 years old. Given the increased life expectancy, elderly patients represent up to $25 \%$ of patients with GBM.

- Age alone is not a predictor of survival in GBM. General condition and performance status strongly influence and guide therapy.

- In this age-group, the biological age is more relevant than the chronological one.

- Surgery aimed at the maximal safe resection, when feasible, followed by adjuvant therapy according to MGMT methylation might be the first therapeutic option. 
- Future clinical trials focusing on GBMs in the elderly subjects could provide more specific data for patient's selection.

\section{Synopsis}

Management of GBM in the elderly population represents a field of growing interest due a longer life expectancy. In this age-group, more than in the young adult, biological age is much more important than chronological one. The date of birth should not exclude $a$ priori access of treatments. Maximal safe resection is proved to be the first option when performance status and general health is good. Adjuvant therapy and decision about management of recurrence should be choose in a multidisciplinary group according to performance of the patients and MGMT methylation. 


\section{Introduction and Background}

Glioblastoma (GBM) is the most common primary malignant tumor of the Central Nervous System (CNS), accounting for 48.3\% of primary malignant brain tumors and $57.3 \%$ of all gliomas. The incidence of GBMs increases with age. The highest rate is recorded in the population between 75 and 84 years old; the disease is two times more frequent in this age range than in the population aged between 55 and 64 years $^{1}$.

Despite advances in surgery and adjuvant treatments, the prognosis remains poor, with a median overall survival of fewer than 18 months in the adult population. An analysis conducted on more than 88000 patients with GBM treated between 2004 and 2013 reports a mild increase in the number of patients surviving three years after the initial diagnosis ${ }^{2}$.

The definition of "elderly" is controversial. The elderly age starts at 65 years old, according to the World Health Organization (WHO); as for GBM patients, the National Comprehensive Cancer Network sets the age to consider a patient into the "elderly category" at 70 years $^{3,4}$. The real-life evidence shows that subjects up to 70 years may still have an active social and intellectual life. Especially in HICs (high-incoming countries), a longer life expectancy is recorded than in the past and or in comparison with LMICs (low and middleincoming countries). In this age range, the physiological age is becoming more relevant than the date of birth to shape the indication and intention to treat.

Age is a negative prognostic factor, and every year of increase in age is associated with a statistically significant decrease in survival in GBM patients ${ }^{4-6}$.

Having this being premised, it is thus mandatory to review the best (current) management of GBMs in the elderly population, since life expectancy (i.e., adding years to life) is growing throughout the world (in Italy life expectancy in 2017 was 83,2 years according to WHO) and elderly patients represent up to $25 \%$ of patients with GBM. In addition, numbers are expected to double in the next two decades $^{7,8}$. 
The management of GBMs is currently multidisciplinary. A balance among available options, such as surgery, chemotherapy, radiation therapy, and experimental approaches, is essential to grant the best feasible outcome and preserve the Quality of Life (i.e., adding life to years, $Q o L)^{9}$. The scope of this review is to report and discuss the state-of-the-art of surgical and adjuvant treatment in elderly patients affected by GBMs.

\section{Current Evidence}

\section{Histomolecular features}

Phenotypical differences across age ranges are only partially explained by differences in known molecular features, such as IDH mutational status, $\mathrm{O}^{6}$-methylguanine-DNA methyl-transferase (MGMT) methylation, and TP53 mutation. IDH mutation, the most important positive prognostic factor in gliomas, is differently expressed in GBM between adult and elderly: IDH1/2 mutations are rarely present in adult GBM and virtually missing in the elderly ${ }^{10-12}$. Although the physiological methylation of cells of the CNS decreases with age, the results of the NOA-08 trial revealed that age does not affect the MGMT promoter methylation frequency. The expression of vascular endothelial growth factor (VEGF) increases in patients older than 55 years old with recurrent GBM ${ }^{13}$. Moreover, TP53 mutation and CDKN1A/p16 alteration are negative markers in patients older than 70 years, conversely than younger subjects ${ }^{11,14}$.

\section{Prognostic Factors}

The first study about prognostic factors employing the recursive partitioning analysis (RPA) was conducted on data collected in the 70s and $80 \mathrm{~s}$, therefore in the pre-Temozolomide (TMZ) era ${ }^{15}$. With a plethora of variables, the authors identified six different classes of risk based on pre- (age, race, gender, KPS, neurological examination, comorbidity, tumor location and size) and post-operative (Histology, EOR, adjuvant therapies) variables, correlating risk classes with oncological outcome. Further updates were released in the following 
years, with fewer prognostic classes (cit.). In the latest edition, the extent of resection and the MGTM promoter methylation were reported as the most relevant prognostic factors for survival in the elderly subjects ${ }^{14,16-20}$.

Along with the previous factors, in elderly a special emphasis should be given to performance status and to general condition as variables strongly influencing and guiding therapies. In this context, the process of selection of those patients who could benefit from treatments, especially surgery, remains a crucial issue. Physiological assessment taking in to account organ function and associated comorbidities, may better predict patient health status than chronological age itself ${ }^{21}$. Elderly patients are not identical and age per se is not a synonymous of frail, that, instead, concerns much more with the physical status of the individual patient. Fraility is generally defined as an unintentional weight loss, self-reported exhaustion, weakness, slow walking speed, and low physical activity ${ }^{22}$. In the elderly patient assessment, surgeons and neuro-oncologists along with the individual patient and family interview, are helped in the decision-making process by the use of scales or questionnaires of evaluation aimed at a more personalized approach ${ }^{11,23-26}$. In addition to the well-known KPS, various scale of fraility have been proposed, , such as the IDAL questionnaire, that assess the ability and motivation to use the phone, to go shopping, or food preparation, to do household work, to take medication, or to use transportation or to handle finances; other are the Oncodage G8 questionnaire, the MMSE or the Chalson Comorbidity Index (CCI) ${ }^{11,27,28}$.

\section{Surgery}

Maximum safe resection is an important prognostic factor in all GBM patients ${ }^{29,30}$. Surgery aims at impacting on the Progression-Free Survival (PFS) and on the Overall Survival (OS) as well as to fulfill other relevant goals such as histological and molecular diagnosis, the relief of the mass effect, the improvement of the neurological status and the reduction of the use of steroids.

Extent of resection is also a prognostic factor for survival in elderly patients.. A recent prospective study conducted on 1452 elderly patients, showed that the craniotomy for tumor resection was a feasible and safe procedure ${ }^{31}$. A pioneering randomized trial analyzed the differential outcome of a group of elderly patients undergoing either surgical resection or biopsy: data showed an improvement in survival of 3 months for the group undergoing resection ${ }^{32}$. Although the difference in survival was small, the randomization design 
made this study a milestone in the field. A later retrospective case-control study demonstrated a gain of $40 \%$ in survival in the resection group (OS 5.7 months vs 4.0 months) compared to the biopsy group ${ }^{33}$. Further retrospective studies confirmed these initial findings in primary GBMs ${ }^{18,34-39}$. A wide metanalysis included a large group of more than 12000 patients older than 60 years, from 34 studies ${ }^{35}$. Patients who underwent to Gross Total Resection (GTR) experienced a gain in OS of 7.05 months on the average, a better functional recovery, a longer PFS, and comparable mortality and morbidity compared to those submitted to biopsy only. A further monoinstitutional retrospective study evaluated the outcome and its associated prognostic factors in 178 elderly subjects, treated from 2004 to 2015. The results confirmed that the elderly population submitted to resection have a statistically significant increase in survival when the complete resection of the contrast-enhancement tumor was achieved, with a 2-years-OS three times longer than that recorded in patients submitted to biopsy alone ${ }^{40}$.

Further studies introduced the concept of EOR thresholds stratification, and assessed the association between the achieved threshold and the outcome: Oszwald showed that a significant increase in survival was observed when the residual volume (RTV) was less than $5 \%$, Pessina et al less than $2 \mathrm{~cm}^{3}$, as absolute value $\mathrm{e}^{40,41}$. Multiples tools are described to increase EOR: intraoperative fluorescence surgery has been recently approved by FDA in USA and it is widely available in neuro-oncological centers. Efficacy on OS of 5-ALA in GBM surgery was first proved with a randomized prospective phase III trial ${ }^{42}$. Despite its widespread use, there are no study in literature focused only on elderly patients ${ }^{43,44}$.

When resection is pushed toward maximal level, the issue of preserving patient integrity is becoming crucial. The use of mapping and monitoring techniques is helpful. There is no dedicated report on the feasibility and safety of awake surgery in the elderly patients; Generally, age is not considered an absolute contraindication for an awake anesthesia, although a strict and careful patient's selection is strongly advised.

Regarding patients' selection, a low pre-operative Karnofsky Performance Status (KPS), a tumor bigger than $4 \mathrm{~cm}$ and the existence of pre-operative deficits (motor, language) have been found to factors negatively influence surgical outcome ${ }^{40,45}$; in particular, the functional status evaluation (KPS), regarding elderly, is the most important prognostic factor and thus represent an essential selection 
criteria $^{17,18,40,46}$. Tab.1 describe the principal studies that investigated oncological outcome of elderly patients after the introduction of Temozolamide (TMZ) in clinical routine; work that compared outcome of biopsy and resection were included.

In patients where stereotaxic biopsy is not feasible due to high risk of complications, advanced MR (perfusion and/or spectroscopy study) or metabolic imaging (11-C-Methionine PET, FET-PET) are recommended ${ }^{47}$.

In the pre-operative stage, it is also important to exclude potential differential diagnosis. To perform a Total Body CT scan with the iodine-contrast agent is advisable to rule out a possible metastatic origin of the brain lesion, or to carefully look at DWI images to exclude infection.

Lastly, surgery (resection or biopsy) has also the goal of providing adequate tissue for complete histo-molecular characterization, considering that the assessment of the MGMT methylation status is incorporated in the clinical routine ${ }^{16,17,48}$ due to its relevant prognostic predictivity, in first-line treatments and potential newer therapeutic regimes ${ }^{49}$.

\section{Adjuvant Treatments}

Several studies support the role of adjuvant treatments in elderly. If evidence about surgery has been available since the early 90 s, data about the safety and efficacy of post-surgical RT in the elderly were published in 2007. The French database (ANOCEF) showed that Radiotherapy (50Gy in $1.8 \mathrm{~Gy}$ fractions) is superior to the best supportive care in elderly patients with a KPS $>/=70$, since it led to an OS of 29.1 vs 16.9 weeks and a PFS of 14.9 vs 5.4 weeks $^{50}$. Initially, the use of hypo-fractionated protocol was reserved to patients with unfavorable prognostic factors defined by age or performance status ${ }^{51}$ to minimize the radiation time exposure. The Canadian Phase

II trial and NORDIC phase III randomized trial demonstrated that a hypo-fractionated regimen is preferable in most cases, both for oncological and functional reasons. The hypo-fractionated schedule is nowadays the standard therapy in patients with unmethylated MGMT promoter. The NORDIC trial showed in patients older than 70y a survival of 7.0 months when the hypo-fractionated protocol was applied and in of 5.2 months when the standard (60 gy in $2 \mathrm{~Gy}$ fractions over 6 weeks) RT was used ${ }^{16}$. 
The NOA-08 trial confirmed these findings, comparing two arms of treatment: RT (60Gy in 30 Fractions) Vs. continuative TMZ (one week on/one week off). RT improved survival in patients with an unmethylated MGMT (PFS 4.6 months in RT group Vs 3.3 months in TMZ group), while TMZ yielded to better PFS in patients with a methylated MGMT promoter (PFS 8.4 months in TMZ group Vs 4.6 in RT group). Both the NORDIC and NOA-08 trials confirmed the prognostic relevance of MGMT methylation status and the consequent use of TMZ, in patients undergone to biopsy or partial resection. However, data on the combination of RT and on the best therapeutic strategy for elderly patients with an unmethylated MGMT promoter are still lacking ${ }^{17}$.

Since the association with short-course radiotherapy proved to be safe and useful in elderly patients, temozolomide became the subject of a further trial (CCTG CE.6/EORTC 26062) that enrolled 281 patients with resectable tumors, in two arms: short-course Radiotherapy ( 15 fractions of $2.67 \mathrm{~Gy}$ each) with temozolomide and up to 12 cycles of maintenance vs exclusive radiotherapy ${ }^{52,53}$. The results favored the chemoradiation arm with an impact on PFS (5.3mo Vs 3.9mo) and on OS (9.3mo Vs 7.6mo). The trial confirmed the importance of MGMT promoter methylation as a favorable prognostic factor; methylated patients had almost a 2-times greater OS than the comparison study arm. The results showed also an advantage in the use of TMZ in the patients with an unmethylated MGMT promoter: OS was 10.0 months Vs 7.9 months.

Globally, radiotherapy is associated with a improvement in OS, (with controversial evidence of cognitive and QoL decline); for this reason short-course of RT delivered by targeted radiation technique is usually recommended. Conversly, TMZ therapy is largely effective and well-tolerated in the elderly, with a rate of severe side effects less than 15\%. TMZ is recommended in patients with methylation of the MGMT promoter following RT. Exclusive TMZ is also an option for patients with a very unfavourable prognosis ${ }^{54}$. While Chemoradiation is used in selected patients with MGMT promoter methylation, the stand-alone hypo-RT treatment or TMZ Chemotherapy is delivered according to MGMT promoter Methylation status. Based on most recent evidences, first line treatments are reported in Figure 1.

Management of Recurrences 
Despite the best optimal multidisciplinary treatments, GBMs inevitably recur and progres. The are no consensus or evidence on choice of the best strategy to apply on recurrence. However, two recent studies ${ }^{55,56}$ proved that any (i.e. Chemotherapy, RT, surgery) treatment is superior (in term of PFS and OS) to support/palliative therapy alone. The most important limitation of those studies was that they did not considered the quality of life of enrolled patients as a goal.

At the best of our knowledge, only two retrospective studies addressed the issue of surgery for recurrent GBM in the elderly. Considering the limitations of the study, such as the selection bias of the population selected for surgery and the small size of the cohort, an improvement in survival of, at least, seven months ${ }^{57}$ was reported in the group who underwent a second surgery for recurrent GBM. A previous work ${ }^{58}$, conversely, did not show any advantage in survival (4 months) in the group of patients that underwent to a secondsurgery; no data about alternative treatments were also reported.

Re-Irradiation (Re-RT) is a well-established option for treatment of recurrent GBM in young adluts also after a second surgery. A recent paper investigated the feasibility and safety of Re-RT in a cohort of elderly patients with good success in term of OS (6.9 months after Re-RT) with only minor side effect ${ }^{59}$. The decision about Re-RT should not be based on age per se.

As a good clinical practice point, by a meticulous patient's selection, evaluating. KPS, previous treatment, tumor volume, location of recurrence, the time between last treatment and recurrence, a surgical removal of the recurrent tumors could be considered with the consensus of a Neuro-Oncological board, while keeping the Quality of Life into account.

\section{Epilepsy and Corticosteroids}

As in the younger population, there is no consensus on the use of AED prophylactic therapy in patients with no history of seizures or on the fast tapering of AEDs when the tumor is stable ${ }^{60,61}$. The onset of seizures can severely compromise the clinical status of an elder patient. The choice of the drug to administer should be done carefully, especially regarding comorbidity or aggressive behavior side effects, along with the compliance on daily drug intake. The starting dose should be lower than in the younger patients, and monotherapy with a "new" AED is usually the first choice ${ }^{48,62}$. About the use of corticosteroids, a consensus agreement advocates keeping the use at 
a minimum. Steroids are usually given to control pre-operative edema, and a rapid tapering is generally recommended in the postoperative period. A plethora of patients receiving a complete resection can accomplish radiotherapy without or with a limited dosage of steroids.

\section{Thromboembolic Event}

GBM is one of the more prothrombotic tumors across all age groups ${ }^{48}$. However, the risk is not age-related as for other diseases. The reported risk is approximately $18 \%$ per year despite pharmacologic thromboprophylaxis. The event is related to decreased mobility, presence of a moderate or severe motor deficit, steroids intake, radiotherapy, and to the disease itself by the release of vasoactive molecules.

For prophylaxis, LMWH is the first choice for its safety profile. A ICV filter might be an option for patients who are suitable for pharmacologic anticoagulation ${ }^{63-66}$.

Timing for prophylaxis after surgery should be individualized, especially in this class of patients. A prompt start (24/48 hours after surgery) of administration of LMWH should be considered. A synergy with hematologists is encouraged to evaluate patients with a high-risk profile in the pre-operative stage, such as those with pre-existing multiple cardio-vascular disease, with previous thrombotic or thromboembolic events and those who take anti-coagulants or anti-platelets drugs to establish the timing of suspension and the better protocol to re-start therapy.

\section{Supportive and Palliative Care}

The end after an oncological disease (i.e. an end of the disease) is a delicate issue. A single surgical manuscript cannot cope with such a delicate complexity. However, it is relevant to observe that the end-of-the-disease issue faces end-of-life issues in the elderly population. In this context, physicians are often asked to answer at some troublesome questions during the initial consultation for an elderly subject with such a lethal form of cancer right from the clinical-radiological presentation of the disease. Addressing these ethical 
aspects with patients and their caregivers is also frequent during the clinical course of the disease. These issues touch each individual in his/her own intimate beliefs and behaviors. Such issues also have a differential understanding and bylaws in different countries and, therefore, result in differential attitudes in approaching and dealing with them. A reasonable despite simplistic advice is to individualize the medical management with the family or caregivers.

\section{Future Directions}

Compared to the younger adult population, the elderly subjects receive less salvage therapies. Bevacizumab, a monoclonal antibody targeting VEGF, is approved for recurrent GBM outside Europe, where may be administered as an off-label regimen. The antibody is not proven to be superior to lomustine ${ }^{67}$ but plays an important role in symptom-relief and steroid-sparing effects ${ }^{68}$. The AVAglio trial investigated the likely efficacy in the elderly, given the significant VEGF overexpression in these age-group tumors. The trial reported a significant increase in the PFS in all patient included, being however the elderly only the $8 \%$ of all sample enrolled. Efficacy and safety in the elderly should be addressed in future trials ${ }^{69}$.

Tumor treating fields (TTFs), instead, represents a novel promising treatment. A randomized phase III trial proved a significantly longer survival in patients receiving TTFs in association with TMZ with a median OS longer of 20 months $^{70}$. Further studies should be performed in the elderly population, assessing compliance in handling the device, cost-effectiveness, and effect after hypo-fractionated radiotherapy.

\section{Conclusion}

Management of GBM in the elderly population represents a field of growing interest due to the epidemiological relevance, increase in time to life and life to years in the general population. Despite the prognosis remains poor, aggressive safe surgical treatment, with brain 
mapping and monitoring techniques can be pursued after careful pre-operative assessment. In this age-group, the biological age is more relevant than the chronological one. A multidisciplinary teamwork is to be encouraged and pursued.

Future clinical trials focusing on GBMs in the elderly patients could provide with more specific data for patient's selection and biomarkers for patients and family counseling about the risk-benefit ratio of the therapeutic management.

\section{Clinics Care Points}

- GBMs in elderly patients represents a field of growing interest due a longer life expectancy

- Age is not per se a contraindication to aggressive treatment as surgery; general clinical condition guides treatment.

- When feasible a Maximum Safe Resection is to address in order to guarantee access to the best treatment options and a longer Overall Survival

- Adjuvant treatments will be based on MGMT methylation statuts, expecially if complete resection could not be achieved

- At Recurrence multiple option can be considered as for young adults.

- More space in future clinical trial should be reserve to elderly patients.

\section{References}

1. Ostrom Quinn T., Cioffi Gino., Gittleman Haley., et al. CBTRUS Statistical Report: Primary Brain and Other Central Nervous 
System Tumors Diagnosed in the United States in 2012-2016. Neuro Oncol 2019;21(5):v1-100. Doi: 10.1093/neuonc/noz150.

2. Zreik Jad., Moinuddin F. M., Yolcu Yagiz U., et al. Improved 3-year survival rates for glioblastoma multiforme are associated with trends in treatment: analysis of the national cancer database from 2004 to 2013. J Neurooncol 2020. Doi: 10.1007/s11060020-03469-w.

3. NCCN Clinical Practice Guidelines in Oncology. Available at: https://www.nccn.org/professionals/physician_gls/default.aspx. Accessed May 31, 2020.

4. Ta Lawrie., Um Ali. Hanna C, Lawrie TA, Rogozińska E, Kernohan A, Je eries S, Bulbeck H, Ali UM, Robinson T, Grant R 2020. Doi: 10.1002/14651858.CD013261.pub2.www.cochranelibrary.com.

5. Thumma Sudheer R., Fairbanks Robert K., Lamoreaux Wayne T., et al. Effect of pretreatment clinical factors on overall survival in glioblastoma multiforme: a Surveillance Epidemiology and End Results (SEER) population analysis. World J Surg Oncol 2012;10. Doi: 10.1186/1477-7819-10-75.

6. Lorimer C. F., Hanna C., Saran F., et al. Challenges to Treating Older Glioblastoma Patients: the Influence of Clinical and Tumour Characteristics on Survival Outcomes. Clin Oncol 2017;29(11):739-47. Doi: 10.1016/j.clon.2017.05.010.

7. Cohen-Inbar Or. Geriatric brain tumor management part II: Glioblastoma multiforme. J Clin Neurosci 2019:1-4. Doi: 10.1016/j.jocn.2019.05.064.

8. Gately Lucy., Collins Anna., Murphy Michael., et al. Age alone is not a predictor for survival in glioblastoma. J Neurooncol 2016;129(3):479-85. Doi: 10.1007/s11060-016-2194-х.

9. Glaser Scott M., Dohopolski Michael J., Balasubramani Goundappa K., et al. Glioblastoma multiforme (GBM) in the elderly: initial treatment strategy and overall survival. J Neurooncol 2017;134(1):107-18. Doi: 10.1007/s11060-017-2493-X.

10. Louis David N., Perry Arie., Reifenberger Guido., et al. The 2016 World Health Organization Classification of Tumors of the Central Nervous System: a summary. Acta Neuropathol 2016:803-20. Doi: 10.1007/s00401-016-1545-1.

11. Wick Antje., Kessler Tobias., Elia Andrew E.H., et al. Glioblastoma in elderly patients: Solid conclusions built on shifting 
sand? Neuro Oncol 2018;20(2):174-83. Doi: 10.1093/neuonc/nox133.

12. Wiestler Benedikt., Claus Rainer., Hartlieb Sabine A., et al. Malignant astrocytomas of elderly patients lack favorable molecular markers: An analysis of the NOA-08 study collective. Neuro Oncol 2013;15(8):1017-26. Doi:

10.1093/neuonc/not043.

13. Nghiemphu P. L., Liu W., Lee Y., et al. Bevacizumab and chemotherapy for recurrent glioblastoma: A single-institution experience. Neurology 2009;72(14):1217-22. Doi: 10.1212/01.wnl.0000345668.03039.90.

14. Arvold Nils D., Reardon David A. Treatment options and outcomes for glioblastoma in the elderly patient. Clin Interv Aging 2014;9:357-67. Doi: 10.2147/CIA.S44259.

15. Curran Walter J., Scott Charles B., Horton John., et al. Recursive partitioning analysis of prognostic factors in three radiation therapy oncology group malignant glioma trials. J Natl Cancer Inst 1993;85(9):704-10. Doi: 10.1093/jnci/85.9.704.

16. Malmström Annika., Grønberg Bjørn Henning., Marosi Christine., et al. Temozolomide versus standard 6-week radiotherapy versus hypofractionated radiotherapy in patients older than 60 years with glioblastoma: The Nordic randomised, phase 3 trial. Lancet Oncol 2012;13(9):916-26. Doi: 10.1016/S1470-2045(12)70265-6.

17. Wick Wolfgang., Platten Michael., Meisner Christoph., et al. Temozolomide chemotherapy alone versus radiotherapy alone for malignant astrocytoma in the elderly: The NOA-08 randomised, phase 3 trial. Lancet Oncol 2012;13(7):707-15. Doi:

10.1016/S1470-2045(12)70164-X.

18. Scott Jacob G., Bauchet Luc., Fraum Tyler J., et al. Recursive partitioning analysis of prognostic factors for glioblastoma patients aged 70 years or older. Cancer 2012;118(22):5595-600. Doi: 10.1002/cncr.27570.

19. Bell Erica Hlavin., Pugh Stephanie L., McElroy Joseph P., et al. Molecular-based recursive partitioning analysis model for glioblastoma in the temozolomide era a correlative analysis based on nrg oncology RTOG 0525. JAMA Oncol 2017;3(6):78492. Doi: 10.1001/jamaoncol.2016.6020.

20. Wick A, Kessler T, Platten M et al. Superiority of Temozolomide Over Radiotherapy for Elderly Patients With RTK II 
Methylation Class, MGMT Promoter-Methylated Malignant Astrocytoma. Neuro Oncol 2020. Doi: 10.1093/neuonc/noaa033.

21. Laigle-Donadey F., Greffard S. Management of glioblastomas in the elderly population. Rev Neurol (Paris) 2020:1-9. Doi: 10.1016/j.neurol.2020.01.362.

22. Fried Linda P., Tangen Catherine M., Walston Jeremy., et al. Frailty in Older Adults: Evidence for a Phenotype. Journals Gerontol Ser A 2001;56(3):M146-57. Doi: 10.1093/gerona/56.3.M146.

23. Lin Hui Shan., Watts J. N., Peel N. M., et al. Frailty and post-operative outcomes in older surgical patients: A systematic review. BMC Geriatr 2016. Doi: 10.1186/s12877-016-0329-8.

24. Rockwood Kenneth., Song Xiaowei., MacKnight Chris., et al. A global clinical measure of fitness and frailty in elderly people. CMAJ 2005;173(5):489-95. Doi: 10.1503/cmaj.050051.

25. Pallis A. G., Ring A., Fortpied C., et al. Eortc workshop on clinical trial methodology in older individuals with a diagnosis of solid tumors. Ann Oncol 2011;22(8):1922-6. Doi: 10.1093/annonc/mdq687.

26. Soubeyran Pierre., Bellera Carine., Goyard Jean., et al. Screening for Vulnerability in Older Cancer Patients: The ONCODAGE Prospective Multicenter Cohort Study. PLoS One 2014;9(12):e115060. Doi: 10.1371/journal.pone.0115060.

27. Di Cristofori Andrea., Zarino Barbara., Fanizzi Claudia., et al. Analysis of factors influencing the access to concomitant chemoradiotherapy in elderly patients with high grade gliomas: role of MMSE, age and tumor volume. J Neurooncol 2017;134(2):377-85. Doi: 10.1007/s11060-017-2537-2.

28. Lawton M Powell., Brody Elaine M. Assessment of Older People: Self-Maintaining and Instrumental Activities of Daily Living 1. n.d.

29. Sanai Nader., Polley Mei Yin., McDermott Michael W., et al. An extent of resection threshold for newly diagnosed glioblastomas: Clinical article. J Neurosurg 2011;115(1):3-8. Doi: 10.3171/2011.2.JNS10998.

30. Molinaro Annette M., Hervey-Jumper Shawn., Morshed Ramin A., et al. Association of Maximal Extent of Resection of Contrast-Enhanced and Non-Contrast-Enhanced Tumor with Survival Within Molecular Subgroups of Patients with Newly 
Diagnosed Glioblastoma. JAMA Oncol 2020;6(4):495-503. Doi: 10.1001/jamaoncol.2019.6143.

31. Schär Ralph T., Tashi Shpend., Branca Mattia., et al. How safe are elective craniotomies in elderly patients in neurosurgery today? A prospective cohort study of 1452 consecutive cases. J Neurosurg 2020;1(aop):1-9. Doi: 10.3171/2020.2.jns193460.

32. Vuorinen V., Hinkka S., Färkkilä M., et al. Debulking or biopsy of malignant glioma in elderly people - A randomised study. Acta Neurochir (Wien) 2003;145(1):5-10. Doi: 10.1007/s00701-002-1030-6.

33. Chaichana Kaisorn L., Garzon-Muvdi Tomas., Parker Scott., et al. Supratentorial glioblastoma multiforme: The role of surgical resection versus biopsy among older patients. Ann Surg Oncol 2011;18(1):239-45. Doi: 10.1245/s10434-010-1242-6.

34. Ewelt Christian., Goeppert Mathias., Rapp Marion., et al. Glioblastoma multiforme of the elderly: The prognostic effect of resection on survival. J Neurooncol 2011;103(3):611-8. Doi: 10.1007/s11060-010-0429-9.

35. Almenawer Saleh A., Badhiwala Jetan H., Alhazzani Waleed., et al. Biopsy versus partial versus gross total resection in older patients with high-grade glioma: A systematic review and meta-analysis. Neuro Oncol 2015;17(6):868-81. Doi: 10.1093/neuonc/nou349.

36. Abdullah K G., Ramayya A., Thawani J P., et al. Factors Associated with Increased Survival after Surgical Resection of Glioblastoma in Octogenarians. PLoS One 2015;10(5):127202. Doi: 10.1371/journal.pone.0127202.

37. Babu Ranjith., Komisarow Jordan M., Agarwal Vijay J., et al. Glioblastoma in the elderly: The effect of aggressive and modern therapies on survival. J Neurosurg 2016:998-1007. Doi: 10.3171/2015.4.JNS142200.

38. Arvold Nils D., Cefalu Matthew., Wang Yun., et al. Comparative effectiveness of radiotherapy with vs. without temozolomide in older patients with glioblastoma. J Neurooncol 2017;131(2):301-11. Doi: 10.1007/s11060-016-2294-7.

39. Noorbakhsh Abraham., Tang Jessica A., Marcus Logan P., et al. Gross-total resection outcomes in an elderly population with glioblastoma: A SEER-based analysis. Clinical article. J Neurosurg 2014;120(1):31-9. Doi: 10.3171/2013.9.JNS13877.

40. Pessina Federico., Navarria Pierina., Cozzi Luca., et al. Is surgical resection useful in elderly newly diagnosed glioblastoma patients? Outcome evaluation and prognostic factors assessment. Acta Neurochir (Wien) 2018;160(9):1779-87. Doi: 
10.1007/s00701-018-3599-4.

41. Oszvald Ági., Güresir Erdem., Setzer Matthias., et al. Glioblastoma therapy in the elderly and the importance of the extent of resection regardless of age: Clinical article. J Neurosurg 2012;116(2):357-64. Doi: 10.3171/2011.8.JNS102114.

42. Stummer Walter., Pichlmeier Uwe., Meinel Thomas., et al. Fluorescence-guided surgery with 5-aminolevulinic acid for resection of malignant glioma: a randomised controlled multicentre phase III trial. Lancet Oncol 2006;7(5):392-401. Doi: 10.1016/S1470-2045(06)70665-9.

43. Stummer Walter., Reulen Hanns Jürgen., Meinel Thomas., et al. Extent of resection and survival in glioblastoma multiforme: Identification of and adjustment for bias. Neurosurgery 2008;62(3):564-74. Doi: 10.1227/01.neu.0000317304.31579.17.

44. Stummer Walter., Tonn Jörg Christian., Mehdorn Hubertus Maximilian., et al. Counterbalancing risks and gains from extended resections in malignant glioma surgery: A supplemental analysis from the randomized 5-aminolevulinic acid glioma resection study: Clinical article. J Neurosurg 2011;114(3):613-23. Doi: 10.3171/2010.3.JNS097.

45. Laws Edward R., Parney Ian F., Huang Wei., et al. Survival following surgery and prognostic factors for recently diagnosed malignant glioma: Data from the glioma outcomes project. J Neurosurg 2003;99(3):467-73. Doi: 10.3171/jns.2003.99.3.0467.

46. Bauchet Luc., Mathieu-Daudé Hélène., Fabbro-Peray Pascale., et al. Oncological patterns of care and outcome for 952 patients with newly diagnosed glioblastoma in 2004. Neuro Oncol 2010;12(7):725-35. Doi: 10.1093/neuonc/noq030.

47. Sciortino Tommaso., Fernandes Bethania., Conti Nibali Marco., et al. Frameless stereotactic biopsy for precision neurosurgery: diagnostic value, safety, and accuracy. Acta Neurochir (Wien) 2019;161(5). Doi: 10.1007/s00701-019-03873-w.

48. Weller Michael., van den Bent Martin., Hopkins Kirsten., et al. EANO guideline for the diagnosis and treatment of anaplastic gliomas and glioblastoma. Lancet Oncol 2014;15(9):395-403. Doi: 10.1016/S1470-2045(14)70011-7.

49. Reardon David A., Brandes Alba A., Omuro Antonio., et al. Effect of Nivolumab vs Bevacizumab in Patients with Recurrent Glioblastoma: The CheckMate 143 Phase 3 Randomized Clinical Trial. JAMA Oncol 2020. Doi:

10.1001/jamaoncol.2020.1024. 
50. Keime-Guibert Florence., Chinot Olivier., Taillandier Luc., et al. Radiotherapy for Glioblastoma in the Elderly. N Engl J Med 2007;356(15):1527-35. Doi: 10.1056/NEJMoa065901.

51. Roa Wilson., Brasher P. M.A., Bauman G., et al. Abbreviated course of radiation therapy in older patients with glioblastoma multiforme: A prospective randomized clinical trial. J Clin Oncol 2004;22(9):1583-8. Doi: 10.1200/JCO.2004.06.082.

52. Perry James R., Laperriere Normand., O’Callaghan Christopher J., et al. Short-Course Radiation plus Temozolomide in Elderly Patients with Glioblastoma. N Engl J Med 2017;376(11):1027-37. Doi: 10.1056/NEJMoa1611977.

53. Perry James R., Laperriere Normand., O’Callaghan Christopher J., et al. A phase III randomized controlled trial of short-course radiotherapy with or without concomitant and adjuvant temozolomide in elderly patients with glioblastoma (CCTG CE.6,

EORTC 26062-22061, TROG 08.02, NCT00482677). J Clin Oncol 2016;34(18_suppl):LBA2-LBA2. Doi: 10.1200/jco.2016.34.18_suppl.lba2.

54. Pérez-Larraya Jaime Gállego., Ducray François., Chinot Olivier., et al. Temozolomide in elderly patients with newly diagnosed glioblastoma and poor performance status: An ANOCEF phase II trial. J Clin Oncol 2011;29(22):3050-5. Doi:

10.1200/JCO.2011.34.8086.

55. Socha Joanna., Kepka Lucyna., Ghosh Sunita., et al. Outcome of treatment of recurrent glioblastoma multiforme in elderly and/or frail patients. J Neurooncol 2016;126(3):493-8. Doi: 10.1007/s11060-015-1987-7.

56. Lombardi Giuseppe., Bellu Luisa., Pambuku Ardi., et al. Clinical outcome of an alternative fotemustine schedule in elderly patients with recurrent glioblastoma: a mono-institutional retrospective study. J Neurooncol 2016;128(3):481-6. Doi:

10.1007/s11060-016-2136-7.

57. Fariña Nuñez Mateo Tomas., Franco Pamela., Cipriani Debora., et al. Resection of recurrent glioblastoma multiforme in elderly patients: a pseudo-randomized analysis revealed clinical benefit. J Neurooncol 2020;146(2):381-7. Doi: 10.1007/s11060-02003393-z.

58. Hager Jasmin., Herrmann Eva., Kammerer Sarah., et al. Impact of resection on overall survival of recurrent Glioblastoma in 
elderly patients. Clin Neurol Neurosurg 2018;174(July):21-5. Doi: 10.1016/j.clineuro.2018.08.033.

59. Straube Christoph., Antoni Stefanie., Gempt Jens., et al. Re-irradiation in elderly patients with glioblastoma: a single institution experience. J Neurooncol 2019;142(2):327-35. Doi: 10.1007/s11060-019-03101-6.

60. Kerrigan Simon., Grant Robin. Antiepileptic drugs for treating seizures in adults with brain tumours. Cochrane Database Syst Rev 2011;(8). Doi: 10.1002/14651858.cd008586.pub2.

61. Tremont-Lukats Ivo., Ratilal B. O., Armstrong T., et al. Antiepileptic drugs for preventing seizures in people with brain tumors. Cochrane Database Syst Rev 2008. Doi: 10.1002/14651858.CD004424.pub2.

62. Rosati Anna., Buttolo Luciano., Stefini Roberto., et al. Efficacy and safety of levetiracetam in patients with glioma: A clinical prospective study. Arch Neurol 2010;67(3):343-6. Doi: 10.1001/archneurol.2009.335.

63. Perry James R. Thromboembolic disease in patients with high-grade glioma. Neuro Oncol 2012;14 Suppl 4(Suppl 4):iv73-80. Doi: 10.1093/neuonc/nos197.

64. Perry J. R., Julian J. A., Laperriere N. J., et al. PRODIGE: A randomized placebo-controlled trial of dalteparin low-molecularweight heparin thromboprophylaxis in patients with newly diagnosed malignant glioma. J Thromb Haemost 2010;8(9):195965. Doi: 10.1111/j.1538-7836.2010.03973.x.

65. Brandes A. A., Scelzi E., Salmistraro G., et al. Incidence and risk of thromboembolism during treatment of high-grade gliomas: A prospective study. Eur J Cancer 1997;33(10):1592-6. Doi: 10.1016/S0959-8049(97)00167-6.

66. Nabi Shahzaib., Kahlon Pushpinderdeep., Bozorgnia Farshid., et al. Predictors of venous thromboembolism in patients with glioblastoma multiforme. J Clin Oncol 2015;33(15_suppl):e13022-e13022. Doi: 10.1200/jco.2015.33.15_suppl.e13022.

67. Wick Wolfgang., Gorlia Thierry., Bendszus Martin., et al. Lomustine and Bevacizumab in Progressive Glioblastoma. N Engl J Med 2017;377(20):1954-63. Doi: 10.1056/NEJMoa1707358.

68. Taal Walter., Oosterkamp Hendrika M., Walenkamp Annemiek M.E., et al. Single-agent bevacizumab or lomustine versus a combination of bevacizumab plus lomustine in patients with recurrent glioblastoma (BELOB trial): A randomised controlled 
phase 2 trial. Lancet Oncol 2014;15(9):943-53. Doi: 10.1016/S1470-2045(14)70314-6.

69. Chinot Olivier L., Wick Wolfgang., Mason Warren., et al. Bevacizumab plus Radiotherapy-Temozolomide for Newly Diagnosed Glioblastoma. N Engl J Med 2014;370(8):709-22. Doi: 10.1056/NEJMoa1308345.

70. Stupp Roger., Taillibert Sophie., Kanner Andrew., et al. Effect of tumor-treating fields plus maintenance temozolomide vs maintenance temozolomide alone on survival in patients with glioblastoma a randomized clinical trial. JAMA - J Am Med Assoc 2017;318(23):2306-16. Doi: 10.1001/jama.2017.18718.

71. BK Kleinschmidt-DeMasters., KO Lillehei., M Varella-Garcia. Glioblastomas in the Older Old. Arch Pathol Lab Med 2005;129(5). Doi: 10.1043/1543-2165(2005)129<0624:GITOO>2.0.CO;2.

72. Combs Stephanie E., Wagner Johanna., Bischof Marc., et al. Postoperative Treatment of Primary Glioblastoma Multiforme With Radiation and Concomitant Temozolomide in Elderly Patients. Int J Radiat Oncol Biol Phys 2008;70(4):987-92. Doi: 10.1016/j.ijrobp.2007.07.2368.

73. Sijben Angelique E., McIntyre John B., Roldán Gloria B., et al. Toxicity from chemoradiotherapy in older patients with glioblastoma multiforme. J Neurooncol 2008;89(1):97-103. Doi: 10.1007/s11060-008-9593-6.

74. Gerstein Johanna., Franz Kea., Steinbach Joachim P., et al. Postoperative radiotherapy and concomitant temozolomide for elderly patients with glioblastoma. Radiother Oncol 2010;97(3):382-6. Doi: 10.1016/j.radonc.2010.06.014.

75. Kimple Randall J., Grabowski Sarah., Papez Michael., et al. Concurrent temozolomide and radiation, a reasonable option for elderly patients with glioblastoma multiforme? Am J Clin Oncol Cancer Clin Trials 2010;33(3):265-70. Doi:

10.1097/COC.0b013e3181a76a24.

76. Lai Rose., Hershman Dawn L., Doan Tieu., et al. The timing of cranial radiation in elderly patients with newly diagnosed glioblastoma multiforme. Neuro Oncol 2010;12(2):190-8. Doi: 10.1093/neuonc/nop004.

77. Laigle-Donadey Florence., Figarella-Branger Dominique., Chinot Olivier., et al. Up-front temozolomide in elderly patients with glioblastoma. J Neurooncol 2010;99(1):89-94. Doi: 10.1007/s11060-009-0110-3. 
78. Kushnir Igal., Tzuk-Shina Tzahalala. Efficacy of Treatment for Glioblastoma Multiforme in Elderly Patients (65+): A Retrospective Analysis. IMAJ 2011.

79. Hashem Sameh A., Salem Ahmed., Al-Rashdan Abdulla., et al. Radiotherapy with concurrent or sequential temozolomide in elderly patients with glioblastoma multiforme. J Med Imaging Radiat Oncol 2012;56(2):204-10. Doi: 10.1111/j.17549485.2011.02325.x.

80. Tanaka Shota., Meyer Fredric B., Buckner Jan C., et al. Presentation, management, and outcome of newly diagnosed glioblastoma in elderly patients. J Neurosurg 2013;118(4):786-98. Doi: 10.3171/2012.10.JNS112268.

81. Fariselli L., Pinzi V., Milanesi I., et al. Short-course radiotherapy in elderly patients with glioblastoma: Feasibility and efficacy of results from a single centre. Strahlentherapie Und Onkol 2013;189(6):456-61. Doi: 10.1007/s00066-013-0346-X.

82. Lee Jeong Heon., Jung Tae Young., Jung Shin., et al. Performance status during and after radiotherapy plus concomitant and adjuvant temozolomide in elderly patients with glioblastoma multiforme. J Clin Neurosci 2013;20(4):503-8. Doi: 10.1016/j.jocn.2012.03.044.

83. Hoffermann Markus., Bruckmann Lukas., Mahdy Ali Kariem., et al. Treatment results and outcome in elderly patients with glioblastoma multiforme - A retrospective single institution analysis. Clin Neurol Neurosurg 2015;128:60-9. Doi: 10.1016/j.clineuro.2014.11.006.

84. Lombardi Giuseppe., Pace Andrea., Pasqualetti Francesco., et al. Predictors of survival and effect of short (40 Gy) or standardcourse (60 Gy) irradiation plus concomitant temozolomide in elderly patients with glioblastoma: a multicenter retrospective study of AINO (Italian Association of Neuro-Oncology). J Neurooncol 2015;125(2):359-67. Doi: 10.1007/s11060-015-1923-x.

85. Welzel Grit., Gehweiler Julian., Brehmer Stefanie., et al. Metronomic chemotherapy with daily low-dose temozolomide and celecoxib in elderly patients with newly diagnosed glioblastoma multiforme: a retrospective analysis. J Neurooncol 2015;124(2):265-73. Doi: 10.1007/s11060-015-1834-x.

86. Karsy Michael., Yoon Nam., Boettcher Lillian., et al. Surgical treatment of glioblastoma in the elderly: the impact of 
complications. J Neurooncol 2018;138(1):123-32. Doi: 10.1007/s11060-018-2777-9.

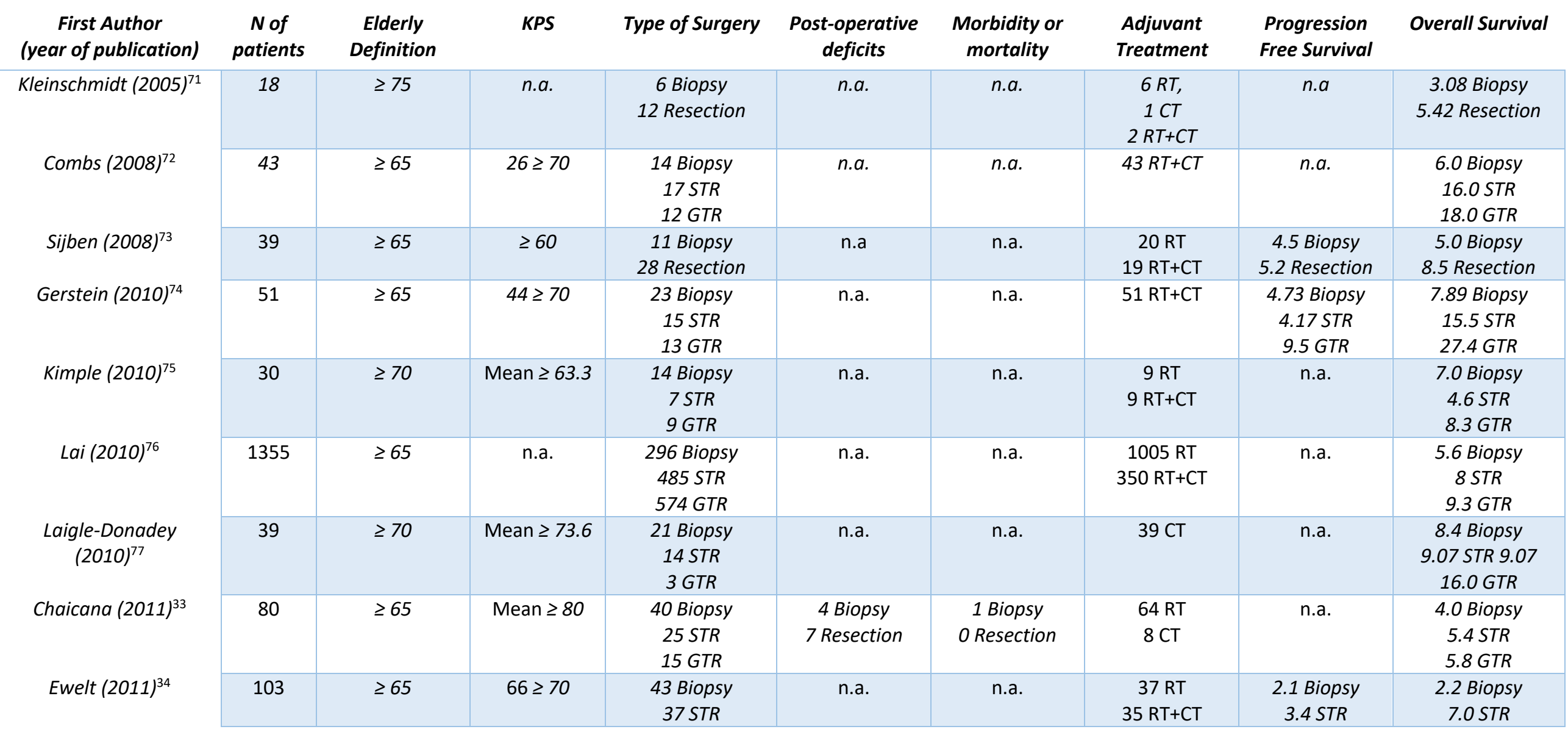




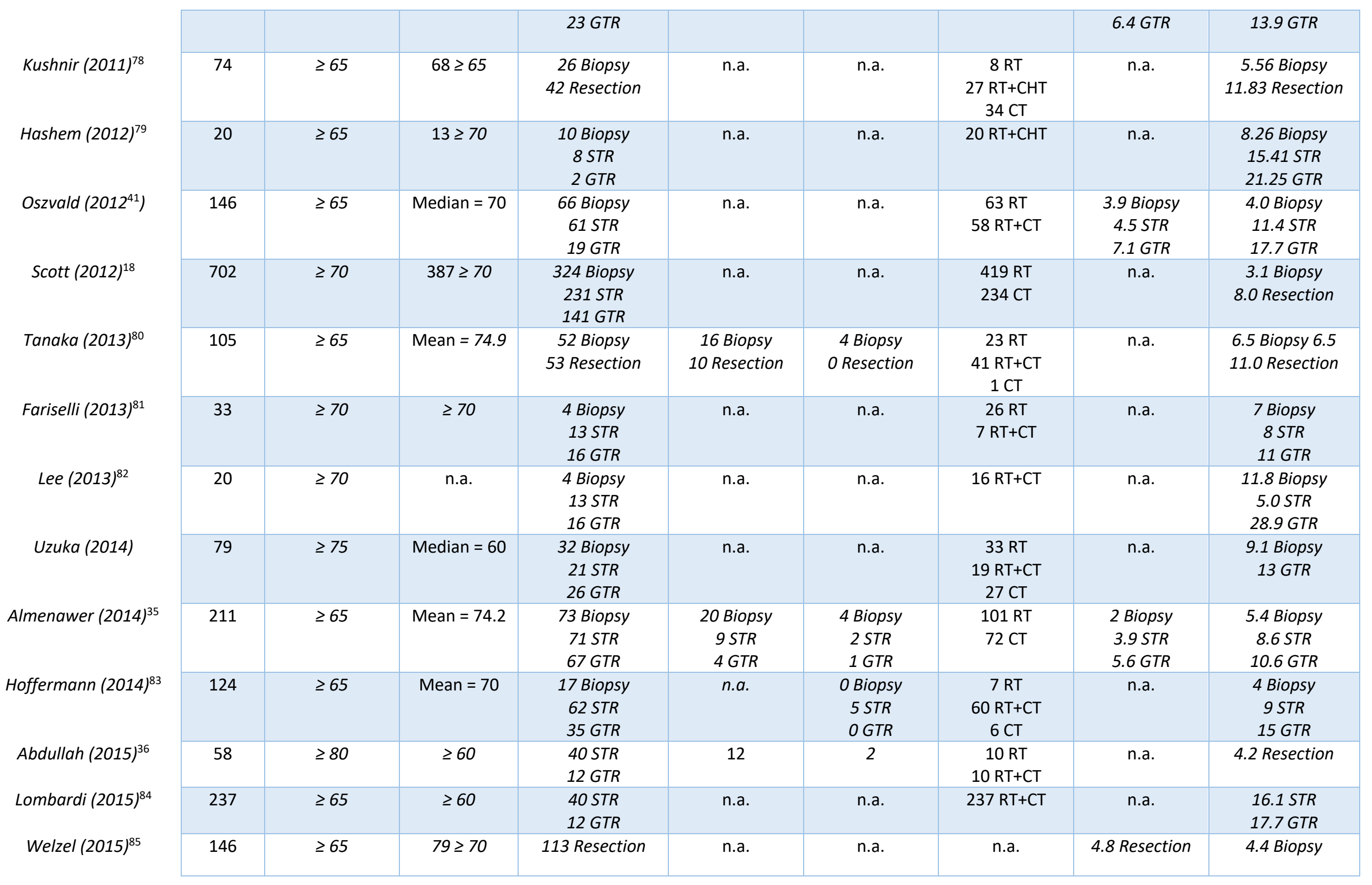




\begin{tabular}{|c|c|c|c|c|c|c|c|c|c|}
\hline & & & & 33 Biopsy & & & & 3.5 Biopsy & 8.1 Resection \\
\hline Babu $(2016)^{37}$ & 120 & $\geq 65$ & Median $=80$ & $\begin{array}{c}63 \text { STR } \\
174 \text { GTR }\end{array}$ & n.a. & n.a. & $110 \mathrm{RT}+\mathrm{CHT}$ & n.a. & $\begin{array}{c}\text { 9.6 STR } \\
14.1 \mathrm{GTR}\end{array}$ \\
\hline Di Cristofori $(2017)^{27}$ & 117 & $\geq 65$ & Median $=70$ & $\begin{array}{l}38 \text { STR } \\
79 \text { GTR }\end{array}$ & 6 Resection & Resection 13 & $\begin{array}{c}84 \mathrm{RT}+\mathrm{CT} \\
16 \mathrm{CT}\end{array}$ & n.a. & $\begin{array}{c}7 \text { STR } \\
11 \text { GTR }\end{array}$ \\
\hline Karsy $(2018)^{86}$ & 82 & $\geq 75$ & Median $=80$ & $\begin{array}{c}18 \text { Biopsy } \\
33 \text { STR } \\
19 \text { GTR }\end{array}$ & $\begin{array}{c}2 \text { Biopsy } \\
5 \text { STR } \\
2 \text { GTR }\end{array}$ & $\begin{array}{c}6 \text { Biopsy } \\
10 \text { STR } \\
6 \text { GTR }\end{array}$ & $\begin{array}{l}32 \mathrm{RT} \\
22 \mathrm{CT}\end{array}$ & n.a. & $\begin{array}{c}\text { 3.7 Biopsy } \\
5 \text { STR } \\
12.1 \text { GTR }\end{array}$ \\
\hline Hager $(2018)^{58}$ & 59 & $\geq 65$ & Median $=90$ & $\begin{array}{c}17 \text { Biopsy } \\
17 \text { STR } \\
25 \text { GTR }\end{array}$ & n.a. & n.a. & $\begin{array}{c}11 \mathrm{RT} \\
41 \mathrm{RT}+\mathrm{CT} \\
25 \mathrm{CT}\end{array}$ & $\begin{array}{l}9.7 \text { Resection } \\
5.6 \text { Biopsy }\end{array}$ & $\begin{array}{l}20.7 \text { Resection } \\
7.4 \text { Biopsy }\end{array}$ \\
\hline Pessina $(2018)^{40}$ & 178 & $\geq 65$ & $142 \geq 70$ & $\begin{array}{c}45 \text { Biopsy } \\
62 \text { STR } \\
63 \text { GTR } \\
8 C R\end{array}$ & $\begin{array}{c}4 \text { Biopsy } \\
4 \text { STR } \\
3 \text { GTR } \\
\text { O CR }\end{array}$ & $\begin{array}{c}1 \text { Biopsy } \\
\text { O STR } \\
2 \text { GTR } \\
\text { OCR }\end{array}$ & $\begin{array}{c}46 \mathrm{RT} \\
132 \mathrm{RT}+\mathrm{CT}\end{array}$ & n.a. & $\begin{array}{c}\text { 8.1 Biopsy } \\
11.9 \text { STR } \\
15.1 \text { GTR } \\
24.5 \text { CR }\end{array}$ \\
\hline
\end{tabular}

Tab.1 The table summarizes the studies comparing oncological outcome of elderly patients that underwent either resection or biopsy after the introduction of Temozolomide. Studies without a report of the Overall Survival (OS) were excluded. Number are reported as absolute value. We report the sample size included, age threshold, KPS (median, mean or majority patients'value), type of surgery, post-operative deficits, post-operative morbidity and mortality, type of adjuvant treatment, months of Progression Free Survival (PFS) and OS. Not avaiable (n.a.) is indicated when the study did not explicitly report the information requested. References are quoted in the main text. 


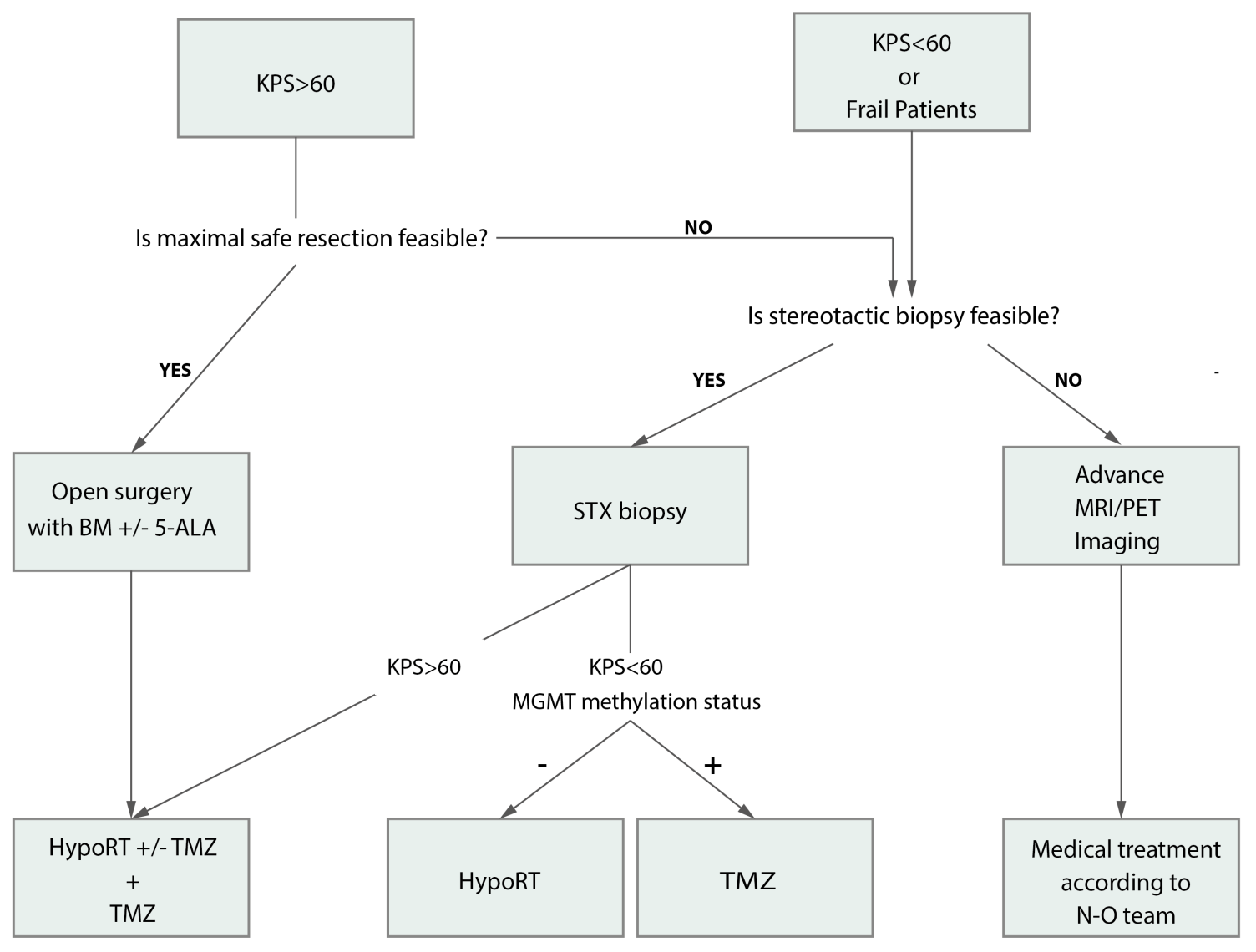

Fig.1 Figure report a flow-chart to an evidence-based algorithm of treatment for elderly patients with GBM. 